\title{
Immune effect of a Newcastle disease virus DNA vaccine with IL-12 as a molecular adjuvant delivered by electroporation
}

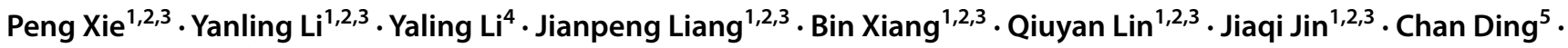 \\ Chenggang $\mathrm{Xu}^{1,2,3} \cdot \operatorname{Tao}^{1,2,3}$
}

Received: 12 December 2019 / Accepted: 17 April 2020 / Published online: 9 June 2020

(c) Springer-Verlag GmbH Austria, part of Springer Nature 2020

\begin{abstract}
Newcastle disease (ND), caused by virulent Newcastle disease virus (NDV) strains, has been one of the most problematic diseases affecting the poultry industry worldwide. Conventional vaccines provide effective protection for birds to survive ND outbreaks, but they may not completely suppress NDV shedding. NDV strains circulate on farms for a long time after the initial infection and cause potential risks. A new vaccine with fast clearance ability and low viral shedding is needed. In this study, we used interleukin-12 (IL-12) as an adjuvant and electroporation (EP) as an advanced delivery system to improve a DNA vaccine candidate. The fusion (F) protein gene from an NDV strain of the prevalent genotype VII.1.1 was cloned to prepare the vaccine. Chickens immunized with the F gene DNA vaccine co-delivered with an IL-12-expressing plasmid DNA showed higher neutralizing antibody levels and stronger concanavalin-A-induced lymphocyte proliferation than those treated with the F gene DNA vaccine alone. The co-delivered vaccine provided $100 \%$ protection, and less viral shedding and a shorter release time were observed in challenged chickens than when the F gene DNA vaccine was administered alone. The use of F gene DNA combined with IL-12 delivered by electroporation is a promising approach for vaccination against ND.
\end{abstract}

\section{Abbreviations \\ APC Antigen-presenting cell \\ CEF Chicken embryo fibroblast}

Handling Editor: William G Dundon.

Peng Xie, Yanling Li and Bin Xiang contributed equally to this work.

$\triangle$ Chenggang Xu

chgangxu@scau.edu.cn

$\triangle$ Tao Ren

rentao6868@126.com

1 College of Veterinary Medicine, South China Agricultural University, Guangzhou, China

2 National and Regional Joint Engineering Laboratory for Medicament of Zoonosis Prevention and Control, Guangzhou, China

3 Key Laboratory of Animal Vaccine Development, Ministry of Agriculture of the People's Republic of China, Guangzhou, China

4 College of Animal Science and Technology, Shihezi University, Shihezi, China

5 Department of Avian Infectious Diseases, Shanghai Veterinary Research Institute, Chinese Academy of Agricultural Sciences, Shanghai, China

$\begin{array}{ll}\text { chIL-12 } & \text { Chicken IL-12 } \\ \text { DMEM } & \text { Dulbecco's modified Eagle's medium } \\ \text { DPC } & \text { Days post-challenge } \\ \text { EP } & \text { Electroporation } \\ \text { F } & \text { Fusion } \\ \text { HN } & \text { Hemagglutinin-neuraminidase } \\ \text { iIFA } & \text { Indirect immunofluorescence assay } \\ \text { IL } & \text { Interleukin } \\ \text { IM } & \text { Intramuscular } \\ \text { ND } & \text { Newcastle disease } \\ \text { NDV } & \text { Newcastle disease virus } \\ \text { Nabs } & \text { Neutralizing antibodies } \\ \text { OD } & \text { Optical density } \\ \text { PBMC } & \text { Peripheral blood mononuclear cell } \\ \text { PCR } & \text { Polymerase chain reaction }\end{array}$

\section{Introduction}

Newcastle disease (ND) has been identified as an important poultry disease by the World Livestock Disease Atlas, 2011. It not only causes severe direct economic losses but also affects humans by decreasing food supplies. The causative agent of ND is avian orthoavulavirus 1, commonly named Newcastle disease virus (NDV), a member of the 
family Paramyxoviridae. Vaccines remain important tools to control the disease in epidemic countries or regions, and co-application of attenuated and killed vaccines has effectively reduced the morbidity and mortality of the disease. However, the failure of vaccination to provide protection against the currently prevalent genotype VII ND has been reported [12]. Mutations occur during the viral replication process, and the natural evolution of wild-type NDV also poses a risk. As of 2019, at least 20 genotypes of NDV in class II and one in class I have been identified, and this number has been increasing [5]. Epidemiology reports have suggested that emerging strains exhibiting antigenic variations may be responsible for recent ND outbreaks in Africa and Asia $[1,20]$. These facts suggest that the conventional vaccine is insufficient for long-term control of ND. Incomplete inactivation of killed NDV vaccine may lead to infections in animals. Studies have found some attenuated vaccines to be contaminated with exogenous viruses, including fowl adenovirus and chicken infectious anemia virus, [19, 31]. In addition, traditional vaccines have the disadvantage of needing to be kept cold during shipping, which is difficult in some developing countries and remote rural areas.

In 1990, DNA was first demonstrated to be effective for producing antigens in vivo [36]. Since then, DNA vaccines have attracted increased interest because of their stability during transport and their safety [6]. DNA vaccines can mimic natural infections, can elicit a broad overall immune response and are stable at ambient temperatures. They can also be produced using polymerase chain reaction (PCR), which makes it easier to match the antigen to epidemic viral strains. In the last decade, a few DNA vaccines have been licensed for use, including vaccines against West Nile virus in horses [3], canine melanoma in dogs [3], and H5N1 avian influenza virus in birds [16]. Due to the inefficiency of direct injection of naked plasmid DNA, delivery methods including the use of electroporation (EP) [25], nanoparticles [9] and biological carriers [10] have been developed to increase the efficiency of uptake of DNA vaccines. By using electrical pulses to generate transient pores in cell membranes, EP allows drugs, RNA, DNA, and proteins to be imported into cells more efficiently than traditional intramuscular (IM) injection $[8,29]$. EP can also enhance vaccine efficacy by eliciting a $\mathrm{CD}^{+} \mathrm{T}$ cell response and inducing a local immune response [4, 26]. Another important strategy for improving DNA vaccines is the use of molecular adjuvants. Cytokines have been used for years as immune adjuvants to improve immune response of vaccines [33]. One of them, IL-12, enhances the proliferation and cytolytic activity of $\mathrm{T}$ and NK cells, activates $\mathrm{T}$ helper 1 cells, and induce the production of interferon- $\gamma$ and other cytokines [23]. IL-12 adjuvant also increases the protective antibody response [23]. Notably, IL-12 can induce strong mucosal immunity, making it especially advantageous in combating respiratory diseases, and it has shown remarkable efficacy in prevention of influenza and pneumococcal disease [2, 22].

The genome of NDV encodes six structural proteins: nucleocapsid protein, matrix protein, phosphoprotein, fusion $(\mathrm{F})$ protein, hemagglutinin-neuraminidase $(\mathrm{HN})$ protein and large polymerase protein. The $\mathrm{F}$ and $\mathrm{HN}$ proteins are naturally expressed on the viral envelope and are recognized first by the immune system during infection. Previous studies have shown that F- or HN-gene-based DNA vaccines can induce the production of neutralizing antibodies (NAbs) in birds that help them to survive NDV infections, and the efficacy of a DNA vaccine based on the $F$ gene has been shown to be superior to that of one based on the HN gene [22,37]. As the use of a vaccine strain homologous to the current circulating viruses is likely to reduce viral shedding [24], we chose the F gene of an NDV strain belonging to genotype VII, which is the prevalent genotype in China, to construct a DNA vaccine. We evaluated the immunogenicity of the F gene DNA vaccine combined with IL-12 adjuvant in chickens and also compared the efficacy of IM injection and EP. The aim of our work was to evaluate a new vaccine candidate for ND control and provide a reference for avian DNA vaccine strategies.

\section{Materials and methods}

\section{Virus, cells, plasmids and chickens}

The NDV GM (Chicken/China/GM/2006; GenBank: DQ486859.1) strain of genotype VII was used for challenge in the animal experiments, and the strain was classified as subgenotype VII.1.1 according to the new classification system reported in 2019 [5]. 293T cells used for transfection were maintained in our laboratory and grown in DMEM (Dulbecco's modified Eagle's medium; Gibco, Thermo Scientific, Waltham, MA, USA) supplemented with $10 \%$ fetal bovine serum (FBS; Gibco) at $37^{\circ} \mathrm{C}$ in $5 \%$ $\mathrm{CO}_{2}$. Primary chicken embryo fibroblast (CEF) cells for the neutralization test were prepared using 9-day-old SPF chicken embryos provided by Da Huanong Animal Health Products Co., Ltd. (Guangdong, China) and were separated as described previously [27]. The eukaryotic expression vector pCAGGS containing a chicken $\beta$-actin promoter was obtained from the Key Laboratory of Animal Vaccine Development in South China Agricultural University. SPF chickens were purchased from Da Huanong Animal Health Products Co., Ltd., and housed in isolators. All animal experiments were approved by the South China Agricultural University Experimental Animal Welfare Ethics Committee. 


\section{Construction of $F$ gene DNA vaccine and chicken IL-12 adjuvants}

RNA was extracted from the allantoic fluid of a chicken embryo inoculated with NDV strain GM and used as the template for $\mathrm{F}$ gene cloning. The RT-PCR products were inserted into the pCAGGS vector to generate pCAG-F. RNA was extracted from peripheral blood mononuclear cells (PBMCs) of leghorn SPF chickens, providing a template for cloning p40 and p35 subunits of chicken IL-12 (chIL-12). The full-length $\mathrm{p} 40$ and $\mathrm{p} 35$ genes were amplified separately by PCR. Then, primer p40-R-L was used with primer $\mathrm{p} 40-\mathrm{F}$ to delete the TGA stop codon and add a part of the linker nucleotide sequence, and the resulting amplicon was $977 \mathrm{bp}$ long. Primer $\mathrm{p} 35-\mathrm{F}-\mathrm{L}$ was also used with primer $\mathrm{p} 35-\mathrm{R}$ to delete the p35 ATG and to add a part of the linker nucleotide sequence, and the resulting amplicon was $645 \mathrm{bp}$ long. Finally, these amplified products were used as templates, and the primers IL-12-F and IL-12-R were used to obtain a fulllength IL-12 gene with the linker SGGGSGGGGSGGGGS between the $\mathrm{p} 40$ and $\mathrm{p} 35$ subunits. Primers were designed based on the reference sequences obtained from the GenBank database (AY262752.1, AY262751.1). The PCR products were cloned into the restriction sites of the pCAGGS vector to generate the construct pCAG-IL-12. The primers used for PCR are listed in Table 1.

\section{In vitro expression from plasmid constructs}

The plasmids pCAGGS, pCAG-F, and pCAG-IL-12 were introduced separately into $293 \mathrm{~T}$ cells by transfection using Lipofectamine $^{\mathrm{TM}} 3000$ (Life Technologies, USA) according to the manufacturer's instructions. Protein expression was detected by indirect immunofluorescence assay (iIFA) after 48 hours. Briefly, the cells were fixed with $4 \%$ paraformaldehyde, permeabilized with $0.1 \%$ Triton $\times-100$, and blocked with PBS containing 5\% bovine serum albumin. The cells were then incubated with a primary rabbit polyclonal antibody against NDV (diluted 1:100 in PBS) or that against IL-12 (diluted 1:100 in PBS) at $37^{\circ} \mathrm{C}$ for $2 \mathrm{~h}$. NDV antibody was prepared by us and stored at the Key Laboratory of Animal Vaccine Development in South China Agricultural University (details not shown). The IL-12 antibody was prepared as follows: Briefly, the chicken IL-12 gene was amplified and cloned into the vector pET-32a (Novagen; EMD-Millipore, Billerica, MA), and the resulting plasmid construct was expressed in Escherichia coli BL21 (DE3) competent cells. The recombinant protein was purified and injected into rabbits to obtain polyclonal serum. FITC-labeled goat antirabbit IgG (BOSTER, Wuhan, China; diluted 1:150 in PBS) was used as the secondary antibody. Fluorescence signals were observed by fluorescence microscopy.

\section{Immunization and challenge experiments}

\section{F gene DNA vaccine administered IM or by EP}

Forty 14-day-old white leghorn SPF chickens were randomly divided into four equal groups $(\mathrm{N}=10)$ for vaccination experiments. For primary immunization, chickens in groups 1,2 , and 3 were inoculated with $100 \mu \mathrm{g}$ of $\mathrm{dd}_{2} \mathrm{O}$, 100- $\mu \mathrm{g}$ pCAGGS, or $100 \mu \mathrm{g}$ of pCAG-F, respectively, by IM injection. Chickens in group 4 were inoculated with $100 \mu \mathrm{g}$ of pCAG-F by EP. EP was performed using an in vivo gene delivery system (TERESA, Shanghai, China) as follows: The vaccine was delivered intramuscularly into the leg using a 1-ml syringe. The syringe was then pulled out, two silver needle electrodes were inserted into the muscles, and six electric pulses of $36 \mathrm{~V}$ (each lasting for $20 \mathrm{~ms}$ ) were immediately delivered around the inoculation site at intervals of $50 \mathrm{~ms}$. After two weeks, the inoculation was repeated as a booster. Three chickens were selected randomly to obtain PBMCs at 14 and 28 days after first immunization. Serum samples were obtained from six randomly selected chickens
Table 1 Primers used for gene amplification

\begin{tabular}{|c|c|c|c|}
\hline Gene & Primer & Sequence of primer $\left(5^{\prime}-3^{\prime}\right)$ & Length (bp) \\
\hline \multirow[t]{2}{*}{$\mathrm{F}$} & GM-F-F & CGGAATTCGCCACCATGGGCTTCAAACCTTCTACC & \multirow[t]{2}{*}{1662} \\
\hline & GM-F-R & CGGCTAGCTCATGCTCTTGTAGTGGCTCT & \\
\hline \multirow[t]{3}{*}{ p40 } & $\mathrm{p} 40-\mathrm{F}$ & ATGTCTCACCTGCTATTTGCCTTAC & \multirow[t]{3}{*}{948} \\
\hline & $\mathrm{p} 40-\mathrm{R}$ & TGATCTGCAAAGCGTGGACCACTC & \\
\hline & p40-R-L & $\begin{array}{l}\text { TCCGCTACCGCCTCCACCAGAGCCTCCTCCACTCTGC } \\
\text { AAAGCGTGGACCACTC }\end{array}$ & \\
\hline \multirow[t]{3}{*}{ p35 } & p35-F-L & $\begin{array}{l}\text { GGTGGAGGCGGTAGCGGAGGCGGAGGGTCGGCAGAG } \\
\text { CACGGCATCGGC }\end{array}$ & \multirow[t]{3}{*}{618} \\
\hline & $\mathrm{p} 35-\mathrm{F}$ & ATGGCAGAGCACGGCATCGGC & \\
\hline & $\mathrm{p} 35-\mathrm{R}$ & TTACATCTCTGCAGTGAGGGCACTCAGG & \\
\hline \multirow[t]{2}{*}{ IL-12 } & IL-12-F & CGGAATTCGCCACCATGTCTCACCTGCTATTTGCCTTAC & \multirow[t]{2}{*}{1608} \\
\hline & IL-12-R & CCCTCGAGTTACATCTCTGCAGTGAGGGCACTCAGG & \\
\hline
\end{tabular}


in each group 28 days after primary immunization. Two weeks after the second immunization, chickens were challenged with NDV at a dose of $10^{3} \mathrm{ELD}_{50}$ in a $200-\mu$ l volume by oculonasal instillation. Oropharyngeal and cloacal swabs were obtained 3, 5, 7, and 9 days post-challenge (DPC) to detect viral shedding. Briefly, swabs were suspended in $1 \mathrm{ml}$ of PBS with antibiotics (penicillin, $4000 \mathrm{U} / \mathrm{ml}$; streptomycin, $2 \mathrm{mg} / \mathrm{ml}$ ) and inoculated into 10-day-old SPF embryonated chicken eggs. The eggs were incubated at $37^{\circ} \mathrm{C}$ for $72 \mathrm{~h}$. The allantoic fluids were collected and tested for the presence of NDV using a standard hemagglutination (HA) inhibition (HI) test. Symptoms and deaths were recorded for 14 days.

\section{F gene DNA vaccine combined with chIL-12 as an adjuvant administered by EP}

An additional 52 14-day-old SPF chickens were randomly divided into four equal groups $(\mathrm{N}=13)$ to investigate the effect of chIL-12 used as an adjuvant to the F gene DNA vaccine. Chickens in group 1 were injected intramuscularly with $\mathrm{ddH}_{2} \mathrm{O}$; those in group 2, with $100 \mu \mathrm{g}$ of pCAG-F; those in group 3, with $100 \mu \mathrm{g}$ of pCAG-F and $100 \mu \mathrm{g}$ of pCAG-IL-12; and those in group 4, with $100 \mu \mathrm{g}$ of pCAGIL-12. All antigens were delivered at a final volume of $100 \mu \mathrm{l}$ by EP as described above. After two weeks, the chickens were administered an equal booster dose of plasmids by the same route. Three chickens were selected randomly to obtain PBMCs at 14 and 28 days after the first immunization. Serum samples were obtained 28 days after the primary immunization from eight randomly selected chickens from each group for Nab detection. Two weeks after the booster, all chickens were challenged with NDV as in the first experiment. Three randomly selected chickens were killed at 3 DPC to measure virus titers in organs as reported previously [13]. Briefly, the collected tissues were homogenized in phosphate-buffered saline containing ampicillin and penicillin. Virus titration was determined in tenfold serial dilutions of supernatant by inoculation into specificpathogen-free embryonated chicken eggs. Virus titers were calculated based on the number of eggs that were positive by hemagglutination at each dilution. Oropharyngeal and cloacal swabs of the remaining 10 chickens in each group were obtained at $3,5,7$, and 9 DPC to detect viral shedding as described previously. All chickens were observed for 14 days, and symptoms and deaths were recorded.

\section{Determination of NAb levels and lymphocyte proliferation}

Various types of antibodies are produced in NDV infection, some of which neutralize the virus, such as antibodies induced by the F protein [28]. In the first and second animal experiments, serum samples from six and eight randomly selected chickens, respectively, from each group were collected after immunization to evaluate the serum antibody response. The virus neutralization test was performed as follows: Heat-inactivated sera were serially diluted twofold and incubated for $2 \mathrm{~h}$ with $100 \mathrm{TCID}_{50}$ of NDV strain GM in DMEM. Serum-NDV mixtures were then applied to CEF monolayers in a 96-well plate with four replicate wells per dilution. After 3 days of incubation, virus was detected in the cell supernatant using a hemagglutination test. A positive HA result indicated that the virus had not been neutralized. The NAb titer was defined as the highest serum dilution at which infectivity was inhibited in $50 \%$ of the wells.

PBMCs were isolated from heparinized peripheral blood from three randomly selected chickens using chicken lymphocyte separation medium (HaoYang, Tianjing, China). Lymphocyte proliferation was determined as reported previously [34]. The isolated cells were washed twice with Hanks' balanced salt solution, washed once in RPMI 1640 (Gibco, USA), and then resuspended in RPMI 1640 containing $10 \%$ FBS, penicillin, and streptomycin at a final concentration of $2 \times 10^{6}$ cells $/ \mathrm{ml}$. The cells were then seeded in 96 -well plates at a density of $2 \times 10^{5}$ cells per well. For the lymphocyte proliferation assay, the cultures were stimulated with $20 \mu \mathrm{l}$ of concanavalin A (ConA, Sigma, $100 \mu \mathrm{g} / \mathrm{ml}$ ) or not stimulated (negative control), both in triplicate, at $39^{\circ} \mathrm{C}$ in $5 \% \mathrm{CO}_{2}$. After $48 \mathrm{~h}, 10 \mu \mathrm{l}$ of MTT $(5 \mathrm{mg} / \mathrm{ml})$ was added to each well, and the incubation was continued for an additional $4 \mathrm{~h}$. After incubation, the supernatant was discarded, and $150 \mu \mathrm{l}$ of dimethyl sulfoxide was added to dissolve the formazan crystals for $10 \mathrm{~min}$. The optical density (OD) of each well was then determined at $570 \mathrm{~nm}$ using a Model 550 Microplate Reader (Bio-Rad Laboratories Co., Ltd., Shanghai, China). The stimulation activity (OD) was calculated using the following formula: $\mathrm{OD}=($ mean $\mathrm{OD}$ of ConA stimulated cells $)-($ mean OD of unstimulated cells).

\section{Statistical analysis}

Statistical analysis was performed using GraphPad Prism 5 software (GraphPad Software Inc., San Diego, CA, USA). All laboratory tests were performed in triplicate, and each value was measured three times. Data are presented as mean values \pm standard deviation (SD). Mean values were analyzed using Student's $t$-test. 


\section{Results}

\section{Eukaryotic expression of the F gene and chIL-12 in vitro}

A 1662-bp fragment of the F gene of NDV and a 1608-bp fragment of the chIL-12 gene were cloned and found to have no mutations. Cells transfected with F and chIL-12 plasmids showed stronger fluorescence than those transfected with the empty vector, indicating that both F protein and chIL-12 were expressed (Fig. 1).

\section{EP is superior to traditional IM injection for $F$ gene DNA vaccine application}

\section{Protective effect and viral shedding}

At 3 DPC, some chickens in the $\mathrm{ddH}_{2} \mathrm{O}$ group and pCAGGS group started showing signs of depression, wryneck, and decreased feeding. A large amount of mucus was observed in the oral cavity when the swabs were collected. At 4 DPC, the chickens started dying, and at 5 DPC, all chickens in both groups were dead. In contrast, two out of ten chickens in the pCAG-F/IM group and one out of ten in the pCAG-F/ EP group began to show minor symptoms at 4 DPC, and only one of them (in the pCAG-F/IM group) died during the experiment (Fig. 2). Viral shedding was monitored by testing oropharyngeal and cloacal swabs obtained on 3, 5, 7 and 9 DPC (Table 2). Virus was detected in $100 \%$ of the

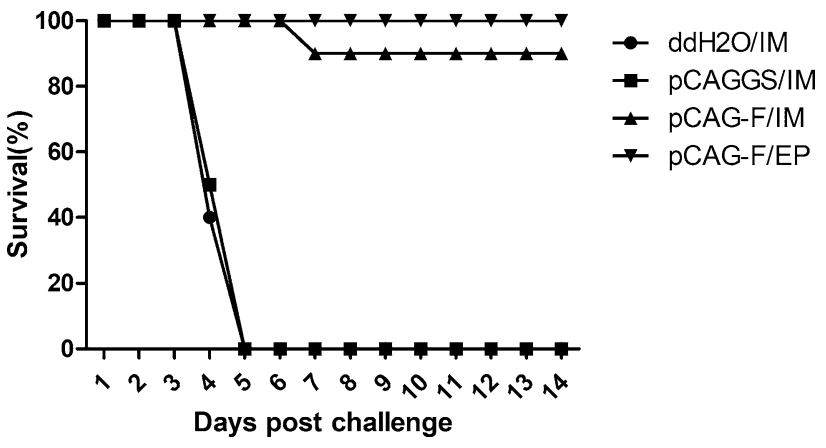

Fig. 2 Protection of chickens by the F gene DNA vaccine delivered by IM injection or EP. Fourteen-day-old SPF chickens $(N=10)$ were inoculated with a DNA vaccine preparation $\left(\mathrm{ddH}_{2} \mathrm{O} / \mathrm{IM}\right.$, pCAGGS/ IM, pCAG-F/IM, or pCAG-F/EP) and boosted after 2 weeks. Two weeks after booster vaccination, the birds were challenged with NDV and mortality for 14 days was evaluated

oropharyngeal and cloacal swabs from chickens in $\mathrm{ddH}_{2} \mathrm{O}$ group and the pCAGGS group at 3 DPC, and by 5 DPC, no chickens in either group had survived. In the pCAG-F/ IM group, viral shedding was detected beginning at 3 DPC, peaked at 5 DPC, and ended at 9 DPC. The pCAG-F/EP group showed a lower shedding rate and shorter shedding duration than the pCAG-F/IM group.

\section{Immune responses after immunization}

The protection and viral shedding rates differed between the pCAG-F/EP and pCAG-F/IM groups, indicating that
Fig. 1 Expression of the $\mathrm{F}$ protein and chIL-12. 293T cells were transfected with pCAGGS (A) or pCAG-F (B) and incubated with polyclonal antibody against NDV or transfected with pCAGGS (C) or pCAGIL-12 (D) and incubated with polyclonal antibody against chIL-12. The photos were taken using a fluorescence microscope to detect fluorescein isothiocyanate signals from the secondary antibody
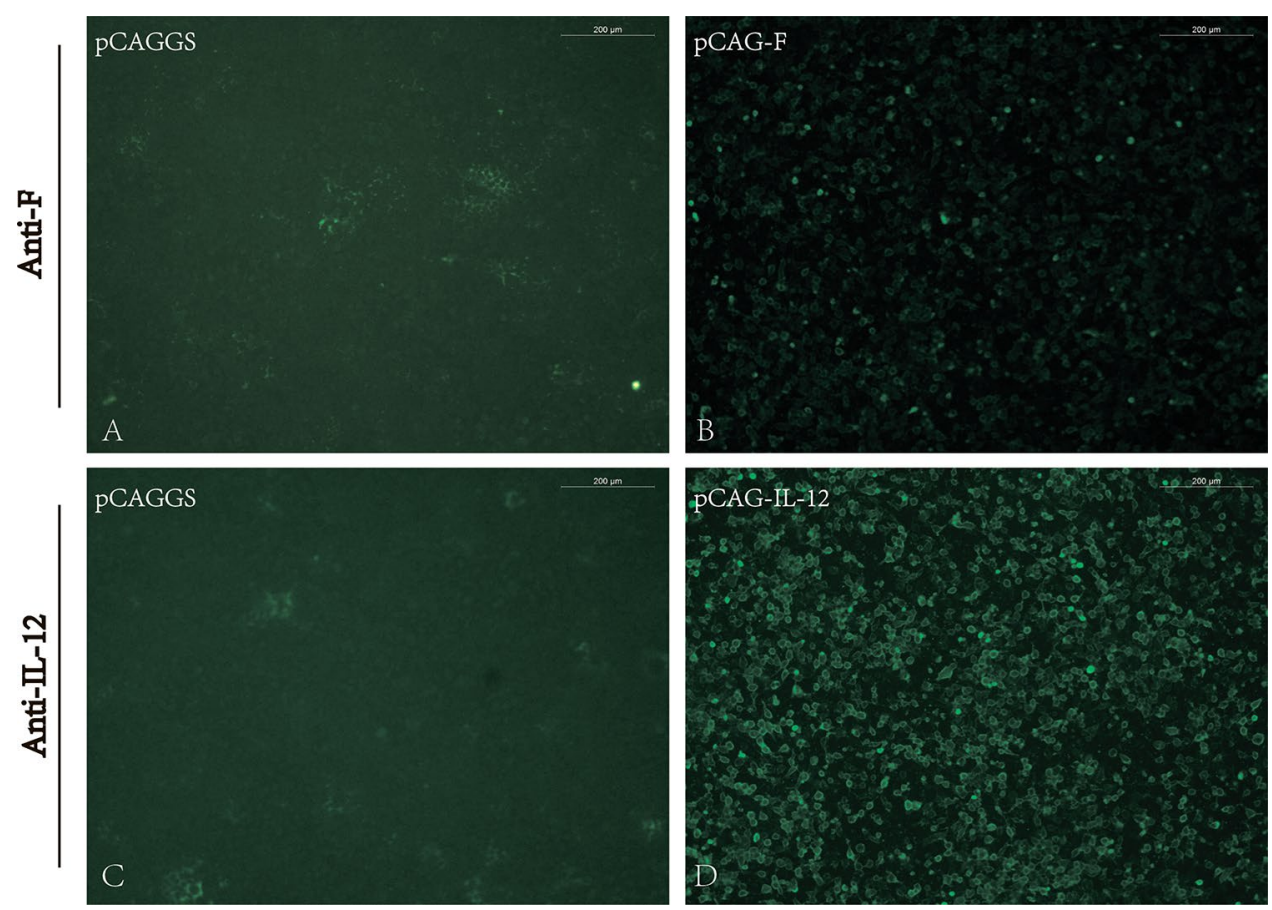
Table 2 Viral shedding after NDV challenge

\begin{tabular}{|c|c|c|c|c|c|c|c|c|}
\hline \multirow[t]{2}{*}{ Group $^{a}$} & \multicolumn{2}{|l|}{$3 \mathrm{DPC}$} & \multicolumn{2}{|c|}{$5 \mathrm{DPC}$} & \multicolumn{2}{|c|}{$7 \mathrm{DPC}$} & \multicolumn{2}{|c|}{$9 \mathrm{DPC}$} \\
\hline & $\mathrm{O}^{\mathrm{b}}$ & $\mathrm{C}^{\mathrm{c}}$ & $\mathrm{O}$ & $\mathrm{C}$ & $\mathrm{O}$ & $\mathrm{C}$ & $\mathrm{O}$ & $\mathrm{C}$ \\
\hline $\mathrm{ddH}_{2} \mathrm{O} / \mathrm{IM}$ & $10 / 10$ & $10 / 10$ & $-{ }^{d}$ & - & - & - & - & - \\
\hline pCAGGS/IM & $10 / 10$ & $10 / 10$ & - & - & - & - & - & - \\
\hline pCAG-F/IM & $2 / 10$ & $0 / 10$ & $7 / 10$ & $6 / 10$ & $6 / 9$ & $3 / 9$ & $3 / 9$ & $2 / 9$ \\
\hline pCAG-F/EP & $0 / 10$ & $1 / 10$ & $4 / 10$ & $2 / 10$ & $2 / 10$ & $2 / 10$ & $0 / 10$ & $0 / 10$ \\
\hline
\end{tabular}

${ }^{\mathrm{a}}$ Chickens in different groups were challenged with $10^{3} \mathrm{ELD}_{50}$ of NDV GM

${ }^{b}$ Oropharyngeal swabs were collected for virus detection

${ }^{\mathrm{c}}$ Cloacal swabs were collected for virus detection

${ }^{\mathrm{d}}$ All birds in the group were dead the two delivery methods might induce different immune responses in chickens. Serum samples were collected 28 days after the first immunization to measure the NAb level. As shown in Fig. 3A, as expected, no antibody was detected in the $\mathrm{ddH}_{2} \mathrm{O}$ and pCAGGS control groups. NAbs were detected in five of the six chickens in pCAG-F/EP group and four of the six chickens in the pCAG-F/IM group, demonstrating that the $\mathrm{F}$ gene DNA vaccine could induce a humoral response. The mean NAb level in the pCAG-F/EP group was higher than that in the pCAG-F/ IM group, indicating that the EP method is superior to IM injection for stimulating DNA-plasmid-induced humoral immunity against the NDV F protein. A lymphocyte proliferation assay was performed to evaluate the ability of lymphocytes from immunized chickens to proliferate after nonspecific stimulation with a mitogen. In this assay, the mean OD value for the pCAG-F/IM group was significantly higher than for the pCAGSS/IM group (Fig. 3B). Notably, the lymphocyte proliferation response for the pCAG-F/EP group was significantly higher than that in the pCAG-F/IM group at 14 or 28 days after primary immunization, suggesting that EP might better sensitize lymphocytes to mitogen-induced proliferation.

\section{ChIL-12 used as an adjuvant enhances the immunogenicity of the NDV F gene DNA vaccine}

\section{Protective effect and viral shedding}

In the $\mathrm{ddH}_{2} \mathrm{O}$ and pCAG-IL-12/EP groups, clinical symptoms occurred at 3 DPC, and members of both groups started dying at 4 DPC (Fig. 4A). Although the mortality in both groups was $100 \%$, the death of chickens in the pCAG-IL-12/ EP group occurred later than in the $\mathrm{ddH}_{2} \mathrm{O}$ group, suggesting that the IL-12 construct alone has an antiviral effect that is, however, not sufficient to protect chickens against fatal NDV infection. All chickens in the pCAG-F/EP and pCAG$\mathrm{F}+\mathrm{pCAG}-\mathrm{IL}-12 / \mathrm{EP}$ groups survived with no clinical signs observed. In the $\mathrm{ddH}_{2} \mathrm{O}$ and pCAG-IL-12 groups, virus was detected in respiratory organs and immune organs, while in the pCAG-F/EP and pCAG-F+pCAG-IL-12/EP groups, no virus was detected (Fig. 4B). Viral shedding lasted until 7 (a)

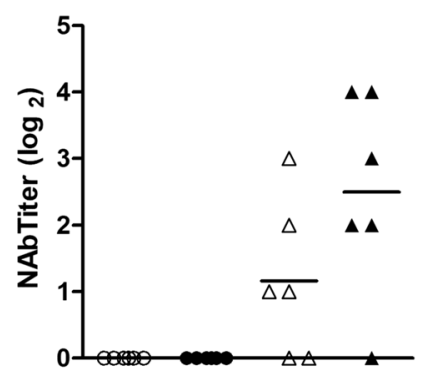

(b)

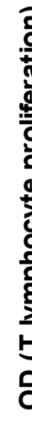

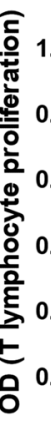

Fig. 3 Immune responses obtained using various plasmid delivery methods. (A) Serum samples were obtained from six randomly selected chickens in each group 28 days after primary immunization. All samples were tested three times on CEF cells to determine the mean NAb titer. Individual values are shown, and solid lines indicate mean values. (B) PBMCs were isolated from three chickens of each group to detect lymphocyte proliferation 14 and 28 days after the first immunization. The error bars indicate the SD. "ns" means no significant difference; $* P<0.05$ 
(a)

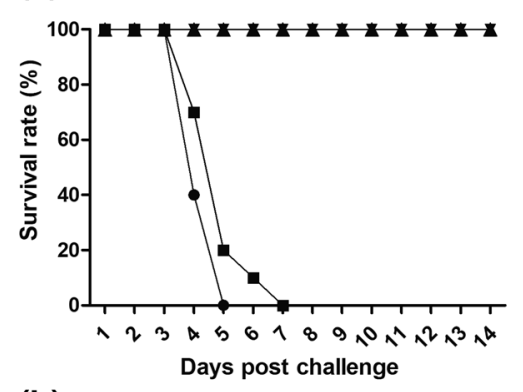

(b)

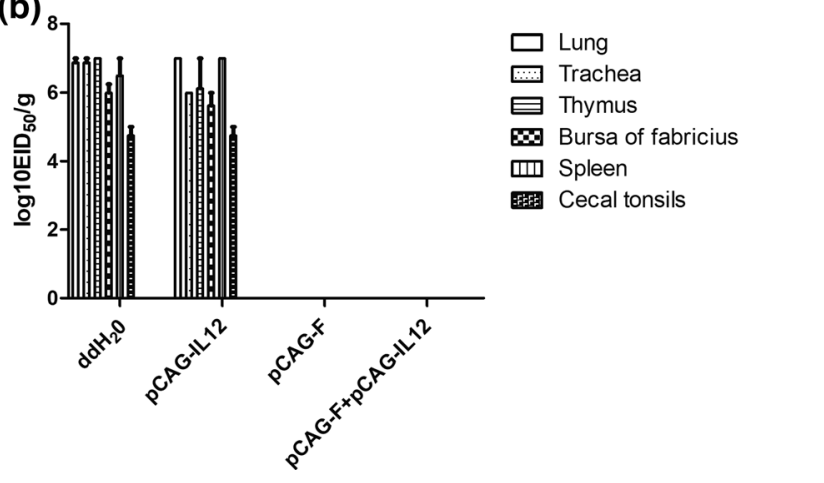

Fig. 4 Protection conferred by pCAG-F and pCAG-IL-12 alone or combined. Fifty-two 14-day-old SPF chickens $(\mathrm{N}=13)$ were inoculated with $\mathrm{ddH}_{2} \mathrm{O} / \mathrm{EP}$, pCAG-IL-12/EP, pCAG-F/EP, or pCAG-F+ pCAG-IL-12 /EP and boosted after 2 weeks. Two weeks after booster vaccination, the birds were challenged with GM. The mortality of ten chickens in each group in 14 days was evaluated (A), and three other chickens were killed and viral titers in different organs were measured by inoculation of 9-day-old embryonated eggs (mean $\pm S D)$. Viral titers in each organ were measured three times (B)

DPC in the pCAG-F/EP group but was only detected at 3 DPC in $10 \%$ of the chickens in the pCAG-F+pCAG-IL-12/ EP group (Table 3).

\section{Immune responses after immunization}

NAbs were detected in all samples from the pCAG-F/EP group except one collected 28 days (Fig. 5A) after primary immunization. The average titer of NAbs in the
pCAG-F+pCAG-IL-12/EP group was higher than that in the pCAG-F/EP group. This suggests that chIL-12 helps the F gene DNA vaccine to stimulate the host immune system, resulting in higher antibody levels. Samples collected from the same group on days 14 and 28 showed no significant differences. The average OD value in the lymphocyte proliferation test was significantly higher for the $\mathrm{pCAG}-\mathrm{F}+\mathrm{pCAG}-$ IL-12/EP group than for the pCAG-F/EP group at 28 days after the primary immunization (Fig. 5B). The average level of lymphocyte proliferation was higher for the pCAG-IL-12/ EP group than for the $\mathrm{ddH}_{2} \mathrm{O} / \mathrm{EP}$ group at 14 and 28 days after the primary immunization.

\section{Discussion}

DNA vaccines have several advantages over conventional vaccines, including increased safety, minimal side effects, stability at ambient temperatures, and the ability to overcome interference by maternal antibodies. However, the low immunogenicity of DNA vaccines are a major barrier to their application; antibody levels and other immune indicators tend to be low after DNA immunization [17]. EP can enhance the uptake of a DNA vaccine by cells by promoting the formation of aqueous pores in cell membranes [15]. EP has been used in several studies to enhance DNA vaccines, and the results have been promising [11]. Oladele and colleagues constructed a DNA vaccine based on the HA gene of $\mathrm{H} 5 \mathrm{~N} 1$ avian influenza virus and found that EP delivery could enhance the antibody response, significantly reduce morbidity, and protect chickens from fatal NDV attack [25]. In this study, we used EP to deliver a DNA vaccine based on the F gene of NDV, and this provided $100 \%$ protection of chickens against virulent NDV challenge, which was better than conventional IM injection (90\%). The average neutralizing antibody level was also higher with EP than with IM injection, indicating that EP administration makes the vaccine more effective.

A lymphocyte proliferation assay is commonly performed to test the nonspecific response of the host after conA
Table 3 Viral shedding after co-delivery of pCAG-F and pCAG-IL-12 via E

\begin{tabular}{|c|c|c|c|c|c|c|c|c|}
\hline \multirow[t]{2}{*}{ Group $^{a}$} & \multicolumn{2}{|l|}{$3 \mathrm{DPC}$} & \multicolumn{2}{|c|}{$5 \mathrm{DPC}$} & \multicolumn{2}{|c|}{$7 \mathrm{DPC}$} & \multicolumn{2}{|c|}{$9 \mathrm{DPC}$} \\
\hline & $\mathrm{O}^{\mathrm{b}}$ & $\mathrm{C}^{\mathrm{c}}$ & $\mathrm{O}$ & $\mathrm{C}$ & $\mathrm{O}$ & $\mathrm{C}$ & $\mathrm{O}$ & $\mathrm{C}$ \\
\hline $\mathrm{ddH}_{2} \mathrm{O} / \mathrm{EP}$ & $10 / 10$ & $10 / 10$ & $-{ }^{d}$ & - & - & - & - & - \\
\hline pCAG-IL-12/EP & $10 / 10$ & $10 / 10$ & $2 / 2$ & $2 / 2$ & - & - & - & - \\
\hline pCAG-F/EP & $1 / 10$ & $0 / 10$ & $3 / 10$ & $2 / 10$ & $2 / 10$ & $2 / 10$ & $0 / 10$ & $0 / 10$ \\
\hline pCAG-F+pCAG-IL-12/EP & $1 / 10$ & $0 / 10$ & $0 / 10$ & $0 / 10$ & $0 / 10$ & $0 / 10$ & $0 / 10$ & $0 / 10$ \\
\hline
\end{tabular}

${ }^{\mathrm{a}}$ Chickens in different groups were challenged with $10^{3} \mathrm{ELD}_{50}$ NDV GM

${ }^{\mathrm{b}}$ Oropharyngeal swabs were collected for virus detection

${ }^{\mathrm{c}}$ Cloacal swabs were collected for virus detection

${ }^{\mathrm{d}}$ All birds in the group were dead 


\section{(a) 28d after First Imunization}
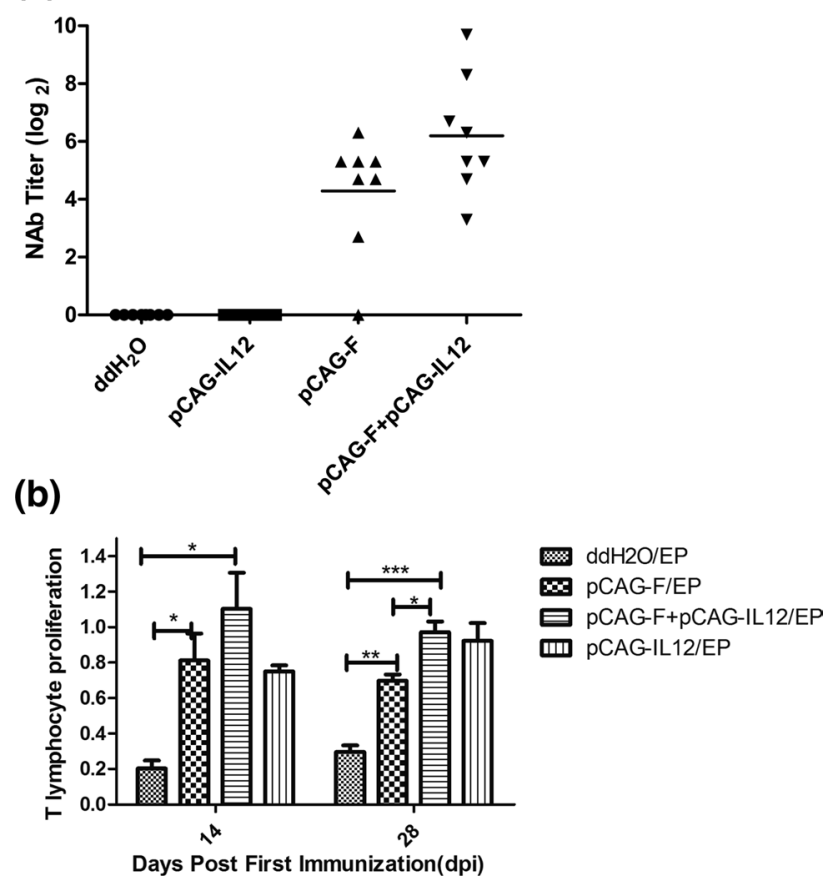

Fig. 5 Immune responses induced by pCAG-F and pCAG-IL-12 alone or combined. (A) Serum samples were obtained 28 days after primary immunization from eight randomly selected chickens from each group. All samples were tested three times on CEF cells to obtain mean NAb titers. (B) PBMCs were isolated from three chickens from each group at 14 and 28 days after the first immunization to measure proliferative activities. The error bars indicate the SD. *, $P<0.05 ; * *, P<0.01 ; * * *, P<0.001$

stimulation. As the nonspecific immune response plays an important role in defense against viral infections, lymphocyte proliferation assays are often performed to evaluate vaccine efficacy, generally together with other methods [34]. At 14 days after priming, the lymphocyte proliferation level of the pCAG-F/EP group was higher than that of the pCAG-F/ IM group, suggesting that the use of EP for delivery resulted in enhanced lymphocyte proliferation. However, since there was no control group vaccinated with the empty plasmid by the EP route, the impact of $\mathrm{F}$ expression on lymphocyte proliferation in this study cannot be evaluated.

The bird in the pCAG-F/IM group that did not survive the virus challenge had no detectable neutralizing antibodies. However, although all of the chickens in the pCAG-F/EP group survived, they did not all have detectable neutralizing antibodies, indicating that antibodies may not be essential for protection and that cell mediated immunity might play an important role in plasmid DNA-induced protection against NDV challenge. Thus, our work demonstrates for the first time that EP can effectively improve the efficacy of NDV DNA vaccines by enhancing the immune response. Other delivery methods, including the use of liposomes, nano-chitosan or Salmonella spp as a vector, have been used to improve NDV DNA vaccines $[7,11]$. A DNA prime-protein booster strategy can further increase the protection rate [14]. A DNA vaccine nano-encapsulated with hollow Ag@ $\mathrm{SiO}_{2}$ nanoparticles stimulates strong mucosal immunity when administered intranasally and has been shown to be protective against NDV. However, this is a high-cost and complicated procedure [38].

Viral shedding is another important indicator for vaccine evaluation. Infected animals that shed virus can become new sources of infection, leading to rapid spreading. It has been reported that chickens inoculated with the commercial La Sota vaccine continue to shed virus for 9 days after challenge with genotype VII NDV, posing a great risk of virus transmission [21]. Both our previous experiments and those of others have shown that the use of a vaccine with higher homology to the challenge NDV strain result in less virus shedding than the use of a heterologous vaccine [24, 32]. In this study, we chose an epidemiologically relevant NDV strain of genotype VII.1.1 to provide the template for the vaccine and for challenge. Although the F gene DNA vaccine delivered by EP could protect chickens from lethal challenge with NDV, the virus was not quickly cleared. Viral shedding still could be detected in oropharyngeal and cloacal swabs of $20 \%$ of the chickens at 7 DPC.

Another strategy to improve the efficacy of DNA vaccines is to add molecular adjuvants. IL-12 is an important member of the interleukin family that is mainly secreted by dendritic cells and monocytes. It is an immunomodulator and a potential adjuvant, since it has been shown to enhance the immunogenicity of vaccines and to induce high-level $\mathrm{CD}^{+}{ }^{+}$and $\mathrm{CD} 8^{+} \mathrm{T}$ cell responses $[18,35]$. To investigate whether chIL-12 has potential as an adjuvant for the NDV F gene DNA vaccine, we constructed an eukaryotic expression plasmid encoding chIL-12 and combined it with the F gene DNA vaccine. Viral shedding was found to be reduced compared with the single DNA vaccine group; only $10 \%$ of the oropharyngeal swabs from chickens were positive for the virus at 3 DPC, and after that, all were negative by virus isolation.

In this study, we verified that the co-administration of chIL-12 and the F gene DNA vaccine resulted in fast clearance of NDV, which would limit the transmission and circulation of the virus in the bird population. Additionally, the mean level of NAb in the pCAG-F + pCAG-IL-12/EP group was higher than that in the pCAG-F group, indicating that chIL-12 also enhances production of specific antibodies. Mitogen-induced lymphocyte proliferation at D28 was also significantly higher for the pCAG-F + pCAG-IL-12/ EP group than for the pCAG-F/EP group, indicating that chIL-12 might enhance lymphocyte proliferation when combined with the F gene DNA vaccine. However, the enhanced lymphocyte proliferation might also have been due to the 
fact that the total dose of plasmid DNA was twice as high in the group receiving both plasmids. The nonspecific mitogen-induced lymphocyte proliferation observed might also have been at least partially induced by pathogen-associated molecular patterns (PAMPs) present in the plasmid DNA preparation, such as LPS or CpG motifs in the plasmid DNA sequence.

Several attempts have been made to improve the efficacy of DNA vaccines by adding molecular adjuvants. Using complement degradation component 3 as an adjuvant has been shown to increase the production of $\operatorname{IgG}$ and $\operatorname{IgA}$ induced by the $\mathrm{F}$ protein DNA vaccine, accompanied by an increased protection rate [39]. Inoculation with IL-18 and F protein together with Salmonella Typhimurium as a vector by the oral route has been shown to increase lymphocyte proliferation, but it does not promote antibody production or provide sufficient protection [7]. Viral shedding was not measured in either of the above-mentioned studies, and therefore, the impact of these approaches on shedding is not known. Recently, another poxvirus-based DNA vaccine targeting the $\mathrm{HN}$ protein and including IL-12 as an adjuvant was evaluated [30]. It was shown that IL-12 can enhance a cell-mediated immune response. However, the vaccine did not provide adequate protection unless another adjuvant, mineral oil, was used simultaneously. Since the amount of plasmid used in that study was much lower than the $100 \mu \mathrm{g}$ used here, and the minimal effective dose of our vaccine has not been determined, further studies are needed to investigate the synergistic effect between the $\mathrm{HN}$ or $\mathrm{F}$ protein and IL-12. Furthermore, since only one strain of epidemic genotype VII was used as challenge virus for vaccine evaluation, the cross-protective effect of the vaccine against other genotypes still needs to be investigated.

In summary, we have evaluated the effects of EP as a delivery method and IL-12 as an adjuvant for NDV DNA vaccines, and the results are promising. DNA vaccines provide a level of safety and flexibility that is not achievable with conventional vaccines. The combination of EP administration and the use of IL-12 as an adjuvant can effectively compensate for the low immunogenicity of the DNA vaccine. Further technological advancements will be needed to further reduce the cost of vaccines for large-scale application in the poultry industry. The current study can provide a reference for research on this and other avian DNA vaccines.

\section{Conclusion}

The F gene DNA combined with chIL-12 significantly enhanced the humoral immune response and lymphocyte proliferation in immunized chickens significantly reduced the rate of virus shedding. This vaccine is a promising new tool for ND control.
Author contributions Peng Xie and Yanling Li contributed equally to this work. PX, Yanling Li, Yaling Li, JL, BX, JJ and QL conducted the experiments. PX and Yanling $\mathrm{Li}$ analyzed the data and wrote the paper. TR, CX and CD designed the experiments and revised the paper. All authors read and approved the final version of the manuscript.

\section{Compliance with ethical standards}

Ethics approval and consent to participate Animals were treated humanely and with regard for alleviation of suffering. All animal experiments were approved by the South China Agricultural University Experimental Animal Welfare Ethics Committee (permit number 2017-V016).

Availability of data and materials The datasets used and/or analyzed during the current study are available from the corresponding author on reasonable request.

Conflict of interest The authors declare that they have no competing interests.

Funding This study was supported by the National Key Research and Development Program of China (no. 2016YFD0501603), National Natural Science Foundation of China (No. 31872492 and no. 31902251), and China Postdoctoral Science Foundation (No. 2018M643107).

\section{References}

1. Abolnik C, Mubamba C, Wandrag DBR, Horner R, Gummow B, Dautu G, Bisschop SPR (2018) Tracing the origins of genotype VIIh Newcastle disease in southern Africa. Transbound Emerg Dis 65:e393-e403

2. Arulanandam BP, O'Toole M, Metzger DW (1999) Intranasal interleukin-12 is a powerful adjuvant for protective mucosal immunity. J Infect Dis 180:940-949

3. Davidson AH, Traub-Dargatz JL, Rodeheaver RM, Ostlund EN, Pedersen DD, Moorhead RG, Stricklin JB, Dewell RD, Roach SD, Long RE, Albers SJ, Callan RJ, Salman MD (2005) Immunologic responses to West Nile virus in vaccinated and clinically affected horses. J Am Vet Med Assoc 226:240-245

4. Dayball K, Millar J, Miller M, Wan YH, Bramson J (2003) Electroporation enables plasmid vaccines to elicit $\mathrm{CD} 8^{+} \mathrm{T}$ cell responses in the absence of $\mathrm{CD}^{+}{ }^{+} \mathrm{T}$ cells. $\mathrm{J}$ Immunol 171:3379-3384

5. Dimitrov KM, Abolnik C, Afonso CL, Albina E, Bahl J, Berg M, Briand FX, Brown IH, Choi KS, Chvala I, Diel DG, Durr PA, Ferreira HL, Fusaro A, Gil P, Goujgoulova GV, Grund C, Hicks JT, Joannis TM, Torchetti MK, Kolosov S, Lambrech B, Lewis NS, Liu HJ, Liu HL, McCullough S, Miller PJ, Monn I, Muller CP, Munir M, Reischak D, Sabra M, Samal SK, Servan de Almeida R, Shittu I, Snoeck CJ, Suarez DL, Van Borm S, Wang Z, Wong FYK (2019) Updated unified phylogenetic classification system and revised nomenclature for Newcastle disease virus. Infect Genet Evol 74:103917

6. Donnelly JJ, Wahren B, Liu MA (2005) DNA vaccines: progress and challenges. J Immunol 175:633-639

7. Gao X, Xu K, Yang G, Shi C, Huang H, Wang J, Yang W, Liu J, Liu Q, Kang Y, Jiang Y, Wang C (2019) Construction of a novel DNA vaccine candidate targeting $\mathrm{F}$ gene of genotype VII Newcastle disease virus and chicken IL-18 delivered by Salmonella. J Appl Microbiol 126:1362-1372 
8. Gardner CL, Trobaugh DW, Ryman KD, Klimstra WB (2016) Electroporation of alphavirus RNA translational reporters into fibroblastic and myeloid cells as a tool to study the innate immune system. Methods Mol Biol 1428:127-137

9. Jazayeri SD, Ideris A, Zakaria Z, Shameli K, Moeini H, Omar AR (2012) Cytotoxicity and immunological responses following oral vaccination of nanoencapsulated avian influenza virus H5 DNA vaccine with green synthesis silver nanoparticles. J Control Release 161:116-123

10. Jazayeri SD, Ideris A, Zakaria Z, Yeap SK, Omar AR (2012) Improved immune responses against avian influenza virus following oral vaccination of chickens with HA DNA vaccine using attenuated Salmonella typhimurium as carrier. Comp Immunol Microbiol Infect Dis 35:417-427

11. Jazayeri SD, Poh CL (2019) Recent advances in delivery of veterinary DNA vaccines against avian pathogens. Vet Res 50:78

12. Ji Y, Liu T, Du Y, Cui X, Yu Q, Wang Z, Zhang J, Li Y, Zhu Q (2018) A novel genotype VII Newcastle disease virus vaccine candidate generated by mutation in the $\mathrm{L}$ and $\mathrm{F}$ genes confers improved protection in chickens. Vet Microbiol 216:99-106

13. Kang Y, Li Y, Yuan R, Feng M, Xiang B, Sun M, Li Y, Xie P, Tan Y, Ren T (2015) Host innate immune responses of ducks infected with Newcastle disease viruses of different pathogenicities. Front Microbiol 6:1283

14. Khulape SA, Maity HK, Pathak DC, Ramamurthy N, Ramakrishnan S, Chellappa MM, Dey S (2019) Evaluation of a fusion gene-based DNA prime-protein boost vaccination strategy against Newcastle disease virus. Trop Anim Health Prod 51:2529-2538

15. Lambricht L, Lopes A, Kos S, Sersa G, Preat V, Vandermeulen G (2016) Clinical potential of electroporation for gene therapy and DNA vaccine delivery. Expert Opin Drug Deliv 13:295-310

16. Lee LYY, Izzard L, Hurt AC (2018) A review of DNA vaccines against influenza. Front Immunol 9:1568

17. Li L, Saade F, Petrovsky N (2012) The future of human DNA vaccines. J Biotechnol 162:171-182

18. Li SS, Kochar NK, Elizaga M, Hay CM, Wilson GJ, Cohen KW, De Rosa SC, Xu R, Ota-Setlik A, Morris D, Finak G, Allen M, Tieu HV, Frank I, Sobieszczyk ME, Hannaman D, Gottardo R, Gilbert PB, Tomaras GD, Corey L, Clarke DK, Egan MA, Eldridge JH, McElrath MJ, Frahm N, Network NHVT (2017) DNA priming increases frequency of T-cell responses to a vesicular stomatitis virus HIV vaccine with specific enhancement of CD8(+) T-cell responses by interleukin-12 plasmid DNA. Clin Vaccine Immunol. https://doi.org/10.1128/CVI.00263-17

19. Li Y, Fu J, Chang S, Fang L, Cui S, Wang Y, Cui Z, Zhao P (2017) Isolation, identification, and hexon gene characterization of fowl adenoviruses from a contaminated live Newcastle disease virus vaccine. Poult Sci 96:1094-1099

20. Liu H, Wang J, Ge S, Lv Y, Li Y, Zheng D, Zhao Y, Castellan D, Wang Z (2019) Molecular characterization of new emerging subgenotype VIIh Newcastle disease viruses in China. Virus Genes 55:314-321

21. Liu J, Zhu J, Xu H, Li J, Hu Z, Hu S, And XW, Liu X (2017) Effects of the $\mathrm{HN}$ antigenic difference between the vaccine strain and the challenge strain of Newcastle disease virus on virus shedding and transmission. Viruses 9:225

22. Lynch JM, Briles DE, Metzger DW (2003) Increased protection against pneumococcal disease by mucosal administration of conjugate vaccine plus interleukin-12. Infect Immun 71:4780-4788

23. Metzger DW (2010) Interleukin-12 as an adjuvant for induction of protective antibody responses. Cytokine 52:102-107

24. Miller PJ, Afonso CL, El Attrache J, Dorsey KM, Courtney SC, Guo Z, Kapczynski DR (2013) Effects of Newcastle disease virus vaccine antibodies on the shedding and transmission of challenge viruses. Dev Comp Immunol 41:505-513

25. Ogunremi O, Pasick J, Kobinger GP, Hannaman D, Berhane Y, Clavijo A, van Drunen Littel-van den Hurk S (2013) A single electroporation delivery of a DNA vaccine containing the hemagglutinin gene of Asian H5N1 avian influenza virus generated a protective antibody response in chickens against a North American virus strain. Clin Vaccine Immunol 20:491-500

26. Peng B, Zhao Y, Xu L, Xu Y (2007) Electric pulses applied prior to intramuscular DNA vaccination greatly improve the vaccine immunogenicity. Vaccine 25:2064-2073

27. Shahsavandi S, Ebrahimi MM, Mohammadi A, Lebas NZ (2013) Impact of chicken-origin cells on adaptation of a low pathogenic influenza virus. Cytotechnology 65:419-424

28. Shahar E, Haddas R, Goldenberg D, Lublin A, Bloch I, Bachner Hinenzon N, Pitcovski J (2018) Newcastle disease virus: is an updated attenuated vaccine needed? Avian Pathol 47:467-478

29. Shi J, Ma Y, Zhu J, Chen Y, Sun Y, Yao Y, Yang Z, Xie J (2018) A review on electroporation-based intracellular delivery. Molecules 23:E3044

30. Su BS, Yin HS, Chiu HH, Hung LH, Huang JP, Shien JH, Lee LH (2011) Immunoadjuvant activities of a recombinant chicken IL-12 in chickens vaccinated with Newcastle disease virus recombinant HN protein. Vet Microbiol 151:220-228

31. Su Q, Li Y, Zhang Y, Zhang Z, Meng F, Cui Z, Chang S, Zhao P (2018) Newcastle disease virus-attenuated vaccine LaSota played a key role in the pathogenicity of contaminated exogenous virus. Vet Res 49:80

32. Sun M, Xiang B, Li Y, Xie P, Gao S, Kang Y, Gao P, Li Y, Wang Z, Liang J, Yu D, Ren T (2017) Generation and evaluation of a genetically attenuated Newcastle disease virus rGM-VIIm as a genotype-matched vaccine. Virus Genes 53:35-43

33. Suschak JJ, Williams JA, Schmaljohn CS (2017) Advancements in DNA vaccine vectors, non-mechanical delivery methods, and molecular adjuvants to increase immunogenicity. Hum Vaccines Immunother 13:2837-2848

34. Wang M, Yang R, Zhang L, Meng X, Fei C, Zhang K, Wang X, Zheng W, Xiao S, Zhang S, Xue F, Hu Y (2014) Sulfated glucan can improve the immune efficacy of Newcastle disease vaccine in chicken. Int J Biol Macromol 70:193-198

35. Wei L, Chu M, Zhang Q, Wang Y, Shang Q, Zhang Y, Zhang G (2014) Interleukin-12 gene adjuvant increases the immunogenicity of virus-like particles of human papillomavirus type 16 regional variant strain. Braz J Infect Dis 18:65-70

36. Wolff JA, Malone RW, Williams P, Chong W, Acsadi G, Jani A, Felgner PL (1990) Direct gene transfer into mouse muscle in vivo. Science 247:1465-1468

37. Zhao K, Zhang Y, Zhang X, Li W, Shi C, Guo C, Dai C, Chen Q, Jin Z, Zhao Y, Cui H, Wang Y (2014) Preparation and efficacy of Newcastle disease virus DNA vaccine encapsulated in chitosan nanoparticles. Int J Nanomedicine 9:389-402

38. Zhao K, Rong G, Hao Y, Yu L, Kang H, Wang X, Wang X, Jin Z, Ren Z, Li Z (2016) IgA response and protection following nasal vaccination of chickens with Newcastle disease virus DNA vaccine nanoencapsulated with $\mathrm{Ag} @ \mathrm{SiO} 2$ hollow nanoparticles. Sci Rep 6:25720

39. Zhao K, Duan X, Hao L, Wang X, Wang Y (2017) Immune effect of Newcastle disease virus DNA vaccine with c3d as a molecular adjuvant. J Microbiol Biotechnol 27:2060-2069

Publisher's Note Springer Nature remains neutral with regard to jurisdictional claims in published maps and institutional affiliations. 